\title{
Empirical Acoustic Attenuation of the Seawater
}

\author{
Xavier Roset-Juan ${ }^{(1)}$, Albert Garcia-Benadí ${ }^{(1)}$, Mike Vanderschaar ${ }^{(2)}{ }^{\text {Joaquín del Río-Fernández }}{ }^{(1)}$, Antoni Mànuel- \\ Làzaro $^{(1)}$ \\ ${ }^{(1)}$ SARTI Research Group. Electronics Department. Universitat Politècnica de Catalunya. \\ ${ }^{(2)}$ LAB Research Group. Universitat Politècnica de Catalunya \\ (1) (2) Rambla de l'Exposició, 24. 08800 Vilanova i la Geltrú. Barcelona. Spain.
}

xavier.roset@upc.edu

\begin{abstract}
This study aims to estimate a model for the underwater acoustic environment in a Mediterranean area and to compare the model with existing other models of the sound attenuation in the zone of interest. The contribution of this paper is to develop a protocol to validate and adjust the offshore underwater attenuation models to a model of a Mediterranean area.
\end{abstract}

Keywords-Sensitivity, GNSS, Hidrophone, attenuation index, seawater acoustic.

\section{INTRODUCTION}

The main surface, about $75 \%$, on the Earth consists of a seawater environment which involves continuous fields of discovery and exploration; nowadays a huge variety of research work takes place in the underwater acoustic environment. Some of these works have been based on either practical or theoretical methods. Shallow waters are characterized by high levels of suspended particles in comparison with the open sea. This can significantly affect the levels of sound attenuation [1]. This study aims to model a Mediterranean area (the western Mediterranean observatory, OBSEA [2]), and to compare the results with two other attenuation estimates at similar sites from literature. The modeling of the environment is a first step to develop different acoustic work in the marine environment, particularly in our test area. One of the objectives pursued is to calibrate a hydrophone offshore, installed in a fixed platform like a cabled underwater observatory as OBSEA. This purpose requires a model of the sound attenuation in the zone of interest. The contribution of this paper is to develop a protocol to validate and adjust the offshore underwater attenuation models to a model of a particular area.

\section{DEVELOPMENT}

In order to adjust the theoretical models of the attenuation underwater sound and the results formulated in [3] [4] [5] [6] to the area of interest, data available from OBSEA cabled seafloor observatory were used: water temperature, pressure, $\mathrm{pH}$ and salinity.

The test was conducted by means of an acoustic source (sound pressure generator, Fig. 1 on surface, and an acoustic receiver on the seafloor at $20 \mathrm{~m}$ deep (hydrophone Fig. 2), set in a permanent underwater observatory (OBSEA). The geolocation of both (source and receiver) was done with a GNSS receiver and raw data was postprocessed with signal phase error correction through reception of data from a ground station. Different acoustics emissions were carried out at different positions. Real time kinematic (RTK) was used to obtain very precise positioning. The RTK system made use of base stations from the ICC broadcast service and the receivers shown in Fig. 3.

A CTD, an AWAC and a pH sensor allowed us to get additional information in order to carry on comparative studies.

\section{A. Equipment}

The equipment that was used is shown in Figures 1, 2 and 3. The sound generator was calibrated with B\&K measurement hydrophone, type 8103, under controlled conditions. The Naxys hydrophone was last calibrated in February 2010 where it showed a $6 \mathrm{~dB}$ loss in sensitivity at low frequencies compared to the previous calibration in June 2008.

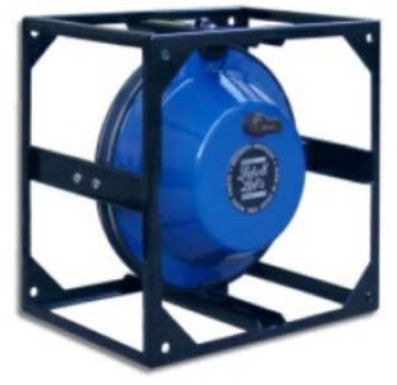

Fig. 1. Lubell sound pressure generator LL9642T model used as acoustic source

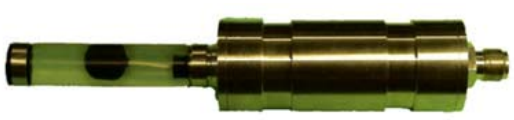

Fig. 2. Hydrophone (Bjørge Naxyx Ethernet Hydrophone 02345 used as acoustic receiver

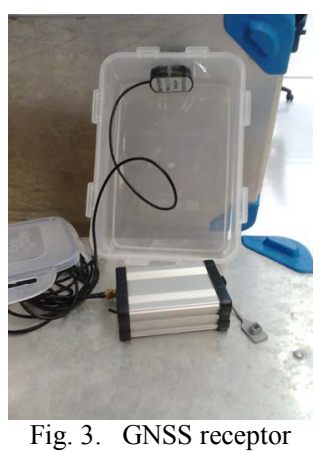




\section{B. Distance evaluation}

Distance is a relevant factor to carry out the measurement. If we are very close the hydrophone cannot get a pure signal reading; however, if we are very far we cannot completely control the trace of the sound in the environment. Thus, the optimal distance of generation has to be tested to ensure minimal influence from reflections from the local environment. These reflections may come from the surface and bottom, but also from other equipment and objects that are present around the hydrophone and the OBSEA platform. Bottom and surface reflection were easiest to theoretically take into account. Fig. 4 shows in detail a test outline.

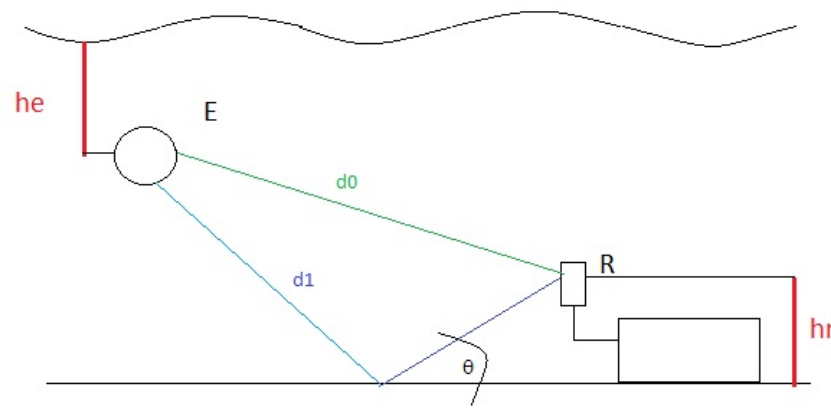

Where

Fig. 4. Test scheme

$\mathrm{hr}=$ The hydrophone distance to the bottom sea.

$\mathrm{d}_{0}=$ The distance from the generator sound to the hydrophone.

$\mathrm{c}=$ The sound velocity in the water.

$\theta=$ The wave reflected angle.

These data show the time interval between the direct reception and the first reflection $(\Delta t),(1)$, according to the rest of the parameters, and also the distance between the generator and the receiver according to the parameters in (2).

$$
\Delta t=\frac{1}{c \cdot \sin \theta} \cdot\left[\left(h r-d_{0} \cdot \sin \theta\right) \pm \sqrt{d_{0}{ }^{2} \cdot \sin ^{2} \theta-h_{r}{ }^{2}}\right]
$$

$$
d 0=\frac{1}{2 \cdot \sin \theta} \cdot\left[\frac{\left(c \cdot \sin \theta \cdot \Delta t-h_{r}\right)^{2}-3 \cdot h_{r}^{2}}{c \cdot \Delta t \cdot \sin \theta-h_{r}}\right]
$$

The sound wave in the environment suffers several energy losses, the first due to the distance covered since the power of the sound wave spreads over the whole wave surface. Another source of loss is due to the sea environment that has a certain absorption detailed in the attenuation coefficient, $\alpha$, which is the value to be estimated here. Contributions to the wave are due to the rebounds on the water surface and on the sea bottom. The relationship between received and transmitted levels is shown in (3); $\mathrm{SL}$ is the source level, $\mathrm{RL}$ is the receipt level and TL is the transmission loss.

$$
R L=S L-T L
$$

The transmission loss model is shown in (4), consisting of a loss component for spreading and a loss component for the absorption; here $\mathrm{r}$ is in kilometers. A preliminary study has been carried out to estimate the power loss due to the wave displacement; to calculate $\mathrm{C}$ where $\mathrm{r}$ is expressed in meters we obtained the result of 17,5. For a cylindrical loss model the constant value is 10 , and 20 for a spherical loss model. The expected value for $\mathrm{C}$ is near 10 since the waters are not very deep; however, the short distance between the generator and the receiver and eliminating reflections in this analysis, the value of $\mathrm{C}$ is closer to spherical loss. To demonstrate that the reflections were not taken into account, in Figure 5 we see the front of one of the signals that was produced by the sound generator.

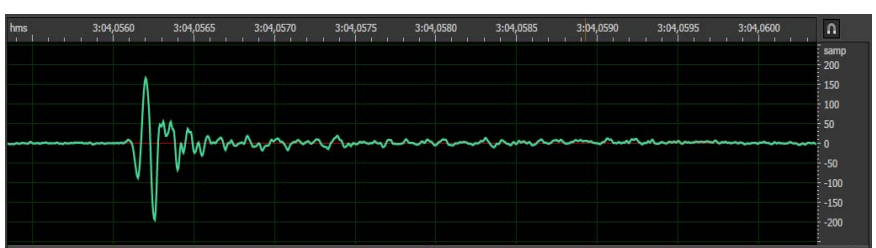

Fig. 5. Received signal

As shown in Fig 5, the main pulse of the signal is clearly defined above the background noise at the hydrophone allowing an accurate estimation of its $136,60 \mathrm{~dB}$ re $1 \mu \mathrm{Pa}$ peak. An echo can be seen mixing with the original signal just after the first main pulse.

$$
T L=C \cdot \log (r \cdot 1000)+\alpha \cdot r
$$

Acoustic emissions were made from two different locations near the hydrophone. The experiment at the first location was made on [2013/03/22-9:28 UTC] at a distance of about $1,2 \mathrm{~km}$ north-west from the hydrophone position; the experiment at the second location was made on [2013/03/2209:59 UTC] and at a distance of $0,9 \mathrm{~km}$ south from the hydrophone position. In Fig. 6 and Fig. 7 the source position at both locations is shown. The movement of the source was due to the vessel drift. The use of RTK allowed making very accurate position estimations

The attenuation estimation is obtained from (5) with units in $\mathrm{dB} / \mathrm{km}$

$$
\alpha=\frac{S L-R L-C \cdot \log (r \cdot 1000)}{r}
$$

SL and RL were measured at the source and receiver respectively and calculated as shown in (6) and (7). The reference pressure $\mathrm{P}_{0}$ is $1 \mu \mathrm{Pa}$. 


$$
\begin{aligned}
& S L=20 \cdot \log \left(\frac{P_{\text {source }}}{P_{0}}\right) \\
& R L=20 \cdot \log \left(\frac{P_{\text {receiver }}}{P_{0}}\right)
\end{aligned}
$$

The sound generator was configured to emit a $10 \mathrm{kHz}$ pulse in manual and automatic mode consisting of 1 and 10 cycles. Sound was generated at continuously changing positions near the hydrophone. The analyzed time interval was comprised within the period where we knew exactly the position of the source.

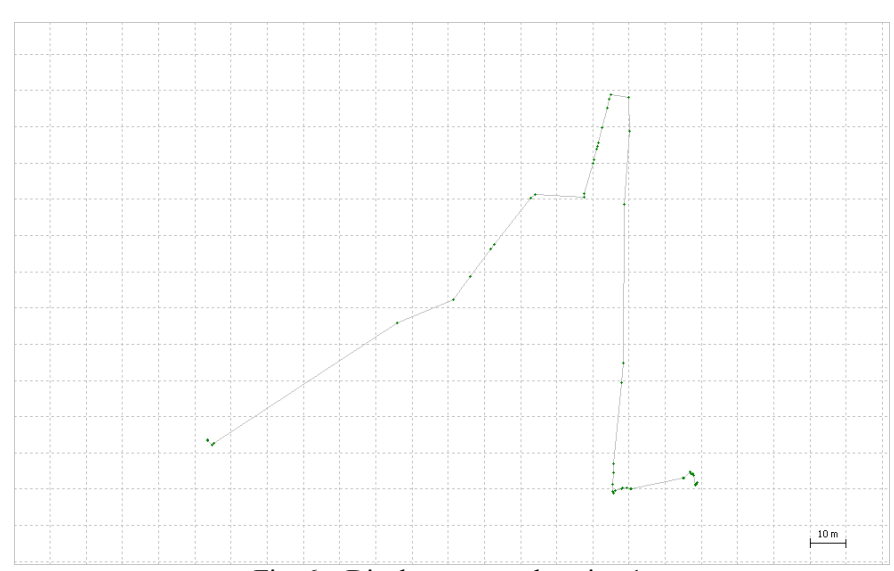

Fig. 6. Displacement at location 1.

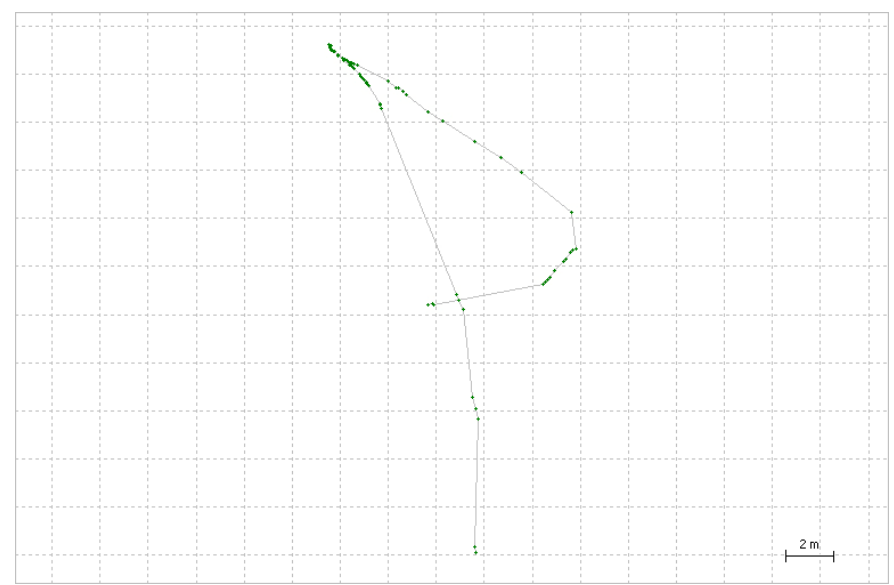

Fig. 7. Displacement at location 2.

\section{RESULTS}

The following Table I shows an example of the position data of fig. 6.

From the two known position interval values we obtained both the hydrophone generated reading and its spectrum, Fig 8 and 9. Both figures show that the location had impulsive background noise very likely produced by crustaceans, typical for shallow water locations. Other noise sources around the hydrophone were the mooring chain and passing ships. It was manually ensured that for the computation of $\alpha$ only those 10
$\mathrm{kHz}$ signals were used that were not contaminated by other noise sources.

TABLE I. POSITION DATA

\begin{tabular}{|c|c|c|c|}
\hline \multirow{2}{*}{$\begin{array}{c}\text { Point } \\
\text { Number }\end{array}$} & \multicolumn{3}{|c|}{ Table Column Head } \\
\cline { 2 - 4 } & Time & $\begin{array}{c}\text { Latitude } \\
(\mathrm{deg})\end{array}$ & $\begin{array}{c}\text { Longitude } \\
(\mathrm{deg})\end{array}$ \\
\hline 1 & $\begin{array}{c}2013 / 03 / 22 \\
09: 59: 33.000\end{array}$ & 41,17460977 & 1,747018135 \\
\hline 2 & $\begin{array}{c}2013 / 03 / 22 \\
09: 59: 35.000\end{array}$ & 41,17461194 & 1,747017418 \\
\hline 3 & $\begin{array}{c}2013 / 03 / 22 \\
10: 00: 01.000\end{array}$ & 41,17465979 & 1,747019249 \\
\hline 4 & $\begin{array}{c}2013 / 03 / 22 \\
10: 00: 03.000\end{array}$ & 41,17466347 & 1,747018068 \\
\hline 5 & $\begin{array}{c}2013 / 03 / 22 \\
10: 00: 07.000\end{array}$ & 41,17466806 & 1,74701621 \\
\hline 6 & $\begin{array}{c}2013 / 03 / 22 \\
10: 00: 38.000\end{array}$ & 41,17470069 & 1,747011897 \\
\hline 7 & $2013 / 03 / 22$ & 41,17470432 & 1,747009527 \\
\hline 8 & $\begin{array}{c}10: 00: 43.000 \\
10: 00: 48.000\end{array}$ & 41,1747065 & 1,747008511 \\
\hline 9 & $2013 / 03 / 22$ & 41,17477609 & 1,746970964 \\
\hline 10 & $\begin{array}{c}10: 03: 02.000 \\
10: 03: 03 / 22\end{array}$ & 41,17477709 & 1,74697074 \\
\hline
\end{tabular}

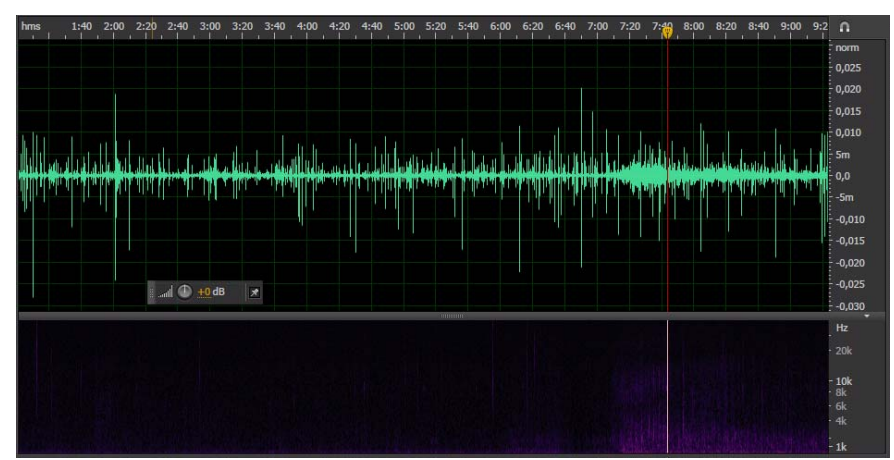

Fig. 8. The hydrophone reception at location 1

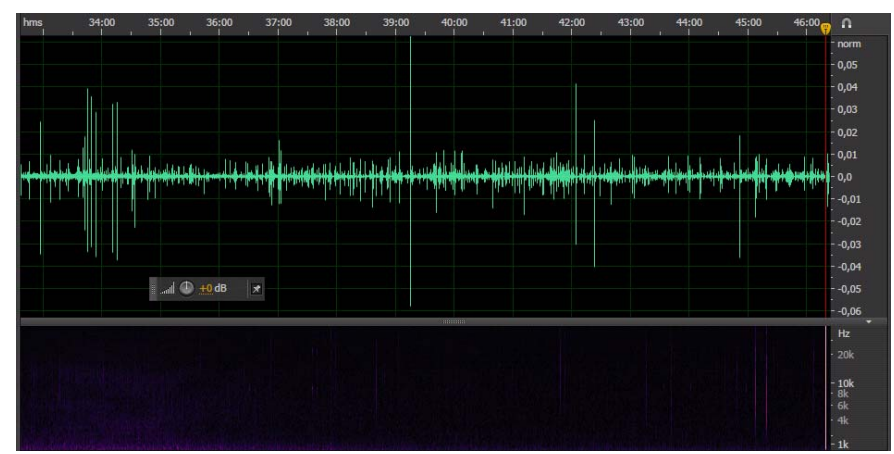

Fig. 9. The hydrophone reception at location 2.

The distance between the points was estimated using the Vicenty[7] formula, since we were positioned at very short distances. The attenuation index was calculated for each source position. A few examples are provided in Table II. 
TABLE II. POSITION RESULTS, PRESSURE RECEIVED

\begin{tabular}{|c|c|c|c|c|}
\hline \multirow{2}{*}{$\begin{array}{c}\text { Point } \\
\text { Number }\end{array}$} & $\begin{array}{c}\mid 4 \\
\text { Distance } \\
(\mathrm{km})\end{array}$ & $\mathrm{P}_{\text {out }}(\mu \mathrm{Pa})$ & $\mathrm{P}_{\text {in }}(\mu \mathrm{Pa})$ & $\alpha$ \\
\hline 1 & 1,227603 & 4860943433 & 6761060 & 2,50 \\
\hline 2 & 1,229305 & 4860943433 & 7283190 & 1,97 \\
\hline 3 & 0,950441 & 4860943433 & 9344510 & 2,32 \\
\hline
\end{tabular}

As a final result and using (5) the attenuation index can be estimated. Table III shows the estimated value of the attenuation index after averaging over all the individual estimations made at each position of the two locations. The value derived from acoustic propagation can be compared to values computed using two models found in literature $[8,9]$. The environmental conditions at OBSEA during the experiments were as follows: $\mathrm{T}=12,88^{\circ} \mathrm{C}, \mathrm{S}=38,1 \% \mathrm{o}, \mathrm{pH}=8,1$ depth $=20 \mathrm{~m}$ (taken from the CTD sensor). The resulting estimates for the attenuation are also shown in Table III. Unfortunately, the theoretical values based on the environmental parameters differ significantly from the one estimated by transmission loss.

TABLE III. COMPARISON BETWEEN ATTENUATION INDICES

\begin{tabular}{|c|c|c|c|}
\hline \multirow{2}{*}{$\begin{array}{c}\text { Frequency } \\
(\mathrm{kHz})\end{array}$} & \multicolumn{3}{|c|}{ Attenuation index $(\mathrm{dB} / \mathrm{km})$} \\
\cline { 2 - 4 } & $\alpha$ & $\alpha[6]$ & $\alpha[9]$ \\
\hline 10 & 2,72 & $9,82 * 10^{-1}$ & $9,78^{*} 10^{-1}$ \\
\hline
\end{tabular}

\section{CONCLUSION}

In this work, a protocol has been assessed to check the sensitivity of a hydrophone without the need to take it out from the sea water. The use of RTK for accurate positioning has worked very well and in theory should have led to a good estimate of the attenuation index. However, the obtained result in Table III is very different from what was expected. Considering the drop in sensitivity of the hydrophone between 2008 and 2010, it is possible that its sensitivity has continued to decrease. A recalibration may be necessary to improve the results. Another important factor that has been detected while carrying out the experiments is the difficulty to control the orientation of the sound source. With the movements of the ship it was not possible to ensure that its orientation towards the hydrophone remained the same throughout the experiment. Another source of error might have been the difficulty to synchronize the clock of the signal generator on the ship and the receiver. Ideally, both of these are synchronized by a GPS, but at the receiver side this is not yet possible with high accuracy. It is foreseen to redo the emission experiments, improving both the transducer orientation and timing synchronization, to see if the attenuation estimate will become more in line with the theoretical value based on environmental parameters.

\section{ACKNOWLEDGMENT}

This work was supported by the Spanish Ministry of Economy and Competitiveness under the research project: "Sistemas Inalambricos para la Extension de Observatorios Submarinos" (CTM2010-15459).

\section{REFERENCES}

[1] S. D. Richards,A. D. Heathershaw, P. D. Thorne."The effect of suspended particulate matter on sound attenuation in seawater". J. Acoust. Soc. Am. 100, September 1996

[2] Aguzzi, J.; Mànuel, A.; Condal, F.; Guillén, J.; Nogueras, M.; Del Rio, J.; Costa, C.; Menesatti, P.; Puig, P.; Sardà, F.; Toma, D.; Palanques, A. "The New Seafloor Observatory (OBSEA) for Remote and Long-Term Coastal Ecosystem Monitoring". Sensors 2011, 11, 5850-5872.

[3] F. H. Fisher and V. P. Simmons "Sound absorption in sea water" J. Acoust. Soc. Am., Vol. 62, No. 3, September 1977

[4] N. P. Fofonoff and A.C. Millard Jr. "Algorithms for computation of fundamental properties of seawater" Unesco/SCOR/ICES/IAPSO Joint Panel on Oceanographic Tables and Standards and SCOR Working Group 51.

[5] V. A. Del Grosso "Tables of the speed of sound in open ocean water (with Mediterranean Sea and Red Sea applicability)" J. Acoust. Soc. Am .Vol.53 N. 5, 1973

[6] Simon D. Richards "The effect of temperature, pressure, and salinity on sound attenuation in turbid seawater" J. Acoust. Soc. Am. Vol.103 N.1, January 1998

[7] T. Vicenty."Direct and inverse solutions of geodesics on the ellipsoid with application of nested equations". Survey Review XXII,176, April 1975

[8] Michael A. Ainslie and James G. McColm." A simplified formula for viscous and chemical absorption in sea". J. Acoust. Soc. Am. 103 (3), March 1998.

[9] R.E. Francois and G. R. Garrison "Sound absorption based on ocean measurements: Part I: Pure water and magnesium sulfate contributions" J. Acoust. Soc. Am. Vol. 72, N.3, December 1982 